\title{
CUSTO DE PRODUÇÃO E SUSTENTABILIDADE DA ALFACE
}

Flávio Alberto Oliva, Maycon Amim Vieira, Pedro Veridiano Baldoto, Ana Paula Pocaya

Universidade do Oeste Paulista - UNOESTE, Faculdade de Ciências Agrárias de Presidente Prudente, Presidente Prudente, SP. E-mail: flavioaoliva@gmail.com

\section{RESUMO}

A produção da alface exige escolhas racionais e utilização eficiente dos fatores produtivos. 0 objetivo deste estudo foi apresentar a apuração de custos e resultados da produção da alface, subsidiando indicadores necessários para tomada de decisão gerencial e estratégia em propriedades rurais. A metodologia recai sobre o entendimento e aplicação dos conceitos e técnicas de mensuração de custos no processo gerencial e custos como ferramenta de controle. Análise da rentabilidade, cálculo dos custos operacionais. As analises demonstraram que os meses quentes e úmidos contribuem para o melhor preço de venda para o produtor, em contrapartida, os meses secos e frios, os preços caem. Diante das inevitáveis oscilações de preços de mercado bem como custo dos insumos, o produtor deve dispor de controles financeiros e informações para reduzir os custos de produção e minimizar riscos ao longo do ano.

Palavras-chave: Produção, Alface, Custo, Resultado, Financeiro.

\section{COST OF PRODUCTION AND SUSTAINABILITY OF LETTUCE}

\begin{abstract}
The production of lettuce requires rational choices and efficient use of production factors. The objective of this study was to present the calculation of costs and lettuce production output, supporting indicators necessary for management decision making and strategy on rural properties. The methodology rests on the understanding and application of concepts and cost measurement techniques in the management process and costs as a control tool. Profitability analysis, calculation of operating costs. The analysis showed that the hot and humid months contribute to the best selling price for the producer, in contrast, dry, cold months, prices fall. Faced with the inevitable market price fluctuations and cost of inputs, the producer must have financial controls and information to reduce costs and minimize risks throughout the year.
\end{abstract}

Keywords: Production, Lettuce, Cost, Result, Financial. 


\section{INTRODUÇÃO}

Originário da Ásia, a alface chegou ao Brasil no século XVI, com os portugueses (IEA, 2005). Cultivada em todas as regiões, e a principal salada consumida pela população, tanto pelo sabor e qualidade nutricional quanto pelo reduzido preço para o consumidor. (RESENDE et al., 2007).

Segundo Sediyama et al. (2007), a alface é consumida na forma de saladas e é fonte de vitaminas $A, B 1, B 2, B 5$ e $C$, fibras e sais minerais, além disso apresenta efeito calmante, diurético e laxante. A especialização crescente do cultivo da alface tem determinado a ampliação da escala de produção.

A evolução de cultivares e sistemas de manejo, tratos culturais, irrigação, espaçamentos, técnicas de colheita e de conservação pós-colheita e mudanças nos hábitos de alimentação impulsionaram o cultivo e tornaram a alface a hortaliça folhosa mais consumida no país (RESENDE et al., 2007).

O Brasil possui uma área de aproximadamente 35.000 hectares plantados com alface, caracterizados pela produção intensiva, pelo cultivo em pequenas áreas e por produtores familiares, gerando cerca de cinco empregos diretos por hectare. (COSTA e SALA, 2005).

A produção da atividade, pelas suas particularidades, exige escolhas racionais e utilização eficiente dos fatores produtivos. Esse processo de tomada de decisão reflete no seu custo total, que por sua vez, impacta os resultados ótimos da atividade (SOUZA et al., 2010).

O cenário atual exige, de técnicos e produtores, cada vez mais informações para a tomada de decisão. Muitas são as variáveis que se deve dominar para reduzir os riscos inerentes à atividade. Para auxiliar na tomada de decisão no cultivo da alface, estudos relacionados a lucratividade e de preços, têm mostrado comportamento oscilante ao durante o ano (MATOS et al., 2008).

Este trabalho tem como objetivo apresentar a apuração de custos e resultados da produção da alface, subsidiando indicadores necessários para tomada de decisão gerencial e estratégia em pequenas e grandes propriedades.

\section{METODOLOGIA}

O estudo propôs-se a contribuir na organização das informações obtidas em planilhas adequadas possibilitando assim a apuração confiável dos custos e das receitas e, consequentemente, proporcionando informações fidedignas para a estruturação de informações necessárias à gestão da cultura da alface. O estudo baseou-se em informações e dados quantitativos do segundo semestre de 2015 e primeiro semestre de 2016, no entanto, possui também caráter qualitativo à medida que se utilizou dessas informações para análises financeiras, econômicas e tomadas de decisões.

\section{RESULTADOS}

Para produzir um hectare de alface, os custos dos insumos totalizaram $\mathrm{R} \$ 12.920,45$ (Tabela 1) sendo sementes $R \$ 1.041,00$, calcário para o condicionamento do solo, $R \$ 558,00$, fertilizantes $R \$ 545,00$, adubo orgânico $R \$ 9.480,00$, fertilizantes de cobertura, $R \$ 810,00$, inseticidas para o controle de pragas, $R \$ 197,00$, fungicida para o controle de doenças $R \$ 271,00$ e $\mathrm{R} \$ 18,00$ com espalhantes adesivos para o melhor aproveitamento dos defensivos (inseticidas e fungicidas) utilizados. 
Tabela 01. Custos dos Insumos por Hectare

\begin{tabular}{lllll}
\hline Insumos & Unidade & Quantidade & Custo Unit. & Custo Total \\
\hline Sementes & $\mathrm{Kg}$ & 5 & $\mathrm{R} \$ 208,25$ & $\mathrm{R} \$ 1.041,25$ \\
Calcário + frete & $\mathrm{T}$ & 3 & $\mathrm{R} \$ 186,00$ & $\mathrm{R} \$ 558,00$ \\
Fertilizantes 4.14.8 & $\mathrm{t}$ & 0,5 & $\mathrm{R} \$ 1.090,00$ & $\mathrm{R} \$ 545,00$ \\
Adubo orgânico & $\mathrm{t}$ & 15 & $\mathrm{R} \$ 632,00$ & $\mathrm{R} \$ 9.480,00$ \\
Fertilizantes de cobertura & $\mathrm{t}$ & 0,6 & $\mathrm{R} \$ 1.350,00$ & $\mathrm{R} \$ 810,00$ \\
Inseticidas & $\mathrm{L}$ & 4 & $\mathrm{R} \$ 49,30$ & $\mathrm{R} \$ 197,20$ \\
Fungicidas & $\mathrm{kg}$ & 5 & $\mathrm{R} \$ 54,20$ & $\mathrm{R} \$ 271,00$ \\
Espalhantes adesivos & $\mathrm{L}$ & 1 & $\mathrm{R} \$ 18,00$ & $\mathrm{R} \$ 18,00$ \\
\hline
\end{tabular}

Total

$\mathrm{R} \$ 12.920,45$

Os custos com os serviços para a produção de um hectare foram calculados em de $\mathrm{R} \$$ 12.140,70 (Tabela 2). Aração, gradagem e aplainamento, $\mathrm{R} \$ 1.004,00$ na aplicação do calcário, $\mathrm{R} \$$ 211,20 , preparação dos canteiros para receber as sementes, $R \$ 1.620,00$, abertura de sulcos e semeadura $\mathrm{R} \$ 810,00$, pulverizações dos defensivos, desbastes, capinas e adubação $\mathrm{R} \$ 2.700,00$, irrigação, $R \$ 1.080,00$, colheita e embalagem para comercialização, $R \$ 3.150,00$, frete $R \$ 400,00$ e $\mathrm{R} \$ 445,50$ com energia elétrica.

Tabela 02 Custos dos Serviços por Hectare

\begin{tabular}{lllll}
\hline Serviços & Unidade & Quantidade & Custo Unit. & Custo Total \\
\hline Aração, gradagem e aplainamento & $\mathrm{h} / \mathrm{m}$ & 8 & $\mathrm{R} \$ 125,50$ & $\mathrm{R} \$ 1.004,00$ \\
Aplicação calcário & $\mathrm{h} / \mathrm{m}$ & 2 & $\mathrm{R} \$ 105,60$ & $\mathrm{R} \$ 211,20$ \\
Prep. Canteiros, aplicação de adubos & $\mathrm{d} / \mathrm{h}$ & 18 & $\mathrm{R} \$ 90,00$ & $\mathrm{R} \$ 1.620,00$ \\
Abertura de sulcos e semeadura & $\mathrm{d} / \mathrm{h}$ & 9 & $\mathrm{R} \$ 90,00$ & $\mathrm{R} \$ 810,00$ \\
Pulverizações & $\mathrm{d} / \mathrm{h}$ & 8 & $\mathrm{R} \$ 90,00$ & $\mathrm{R} \$ 720,00$ \\
Desbastes, capinas, adubação cob. & $\mathrm{d} / \mathrm{h}$ & 30 & $\mathrm{R} \$ 90,00$ & $\mathrm{R} \$ 2.700,00$ \\
Irrigação & $\mathrm{d} / \mathrm{h}$ & 12 & $\mathrm{R} \$ 90,00$ & $\mathrm{R} \$ 1.080,00$ \\
Colheita e embalagem & $\mathrm{d} / \mathrm{h}$ & 35 & $\mathrm{R} \$ 90,00$ & $\mathrm{R} \$ 3.150,00$ \\
Frete & & & & $\mathrm{R} \$ 400,00$ \\
Energia Elétrica & & & & $\mathrm{R} \$ 445,50$ \\
\hline Total & & & $\mathrm{R} \$ \mathbf{1 2 . 1 4 0 , 7 0}$
\end{tabular}

O custo de produção de um hectare de alface, incluindo insumos de $\mathrm{R} \$ 12.920,45$ (Tabela 1) e $R \$ 12.140,70$ de serviços (Tabela 2 ), totalizou $R \$ 25.061,15$. A caixa da alface custa $R \$ 16,15$ de insumos e $\mathrm{R} \$ 15,18$ de serviços, num total de $\mathrm{R} \$ 31,33$. Por fim, o custo de produção de um $\mathrm{Kg}$ da alface foi de $\mathrm{R} \$ \mathbf{0 , 6 5}$ de insumos e $\mathrm{R} \$ 0,58$ de serviços, totalizando $\mathrm{R} \$ 1,23$ por $\mathrm{Kg}$ do produto produzido.

Tabela 03. Custos Totais de Produção da Alface

\begin{tabular}{llll}
\hline Custos & Custo/ha & Custo/Caixa & Custo/Kg \\
\hline Insumos & $\mathrm{R} \$ 12.920,45$ & $\mathrm{R} \$ 16,15$ & $\mathrm{R} \$ 0,65$ \\
Serviços & $\mathrm{R} \$ 12.140,70$ & $\mathrm{R} \$ 15,18$ & $\mathrm{R} \$ 0,58$ \\
\hline Total & $\mathrm{R} \mathbf{2 5 . 0 6 1 , 1 5}$ & $\mathrm{R} \$ \mathbf{3 1 , 3 3}$ & $\mathbf{R} \mathbf{1 , 2 3}$ \\
\hline
\end{tabular}

Sabe-se que assim como qualquer outra cultura, os preços pagos aos produtores pelos produtos produzidos, oscilam muito durante o ano até mesmo dentro de um semestre ou 
até dentro do mesmo mês. A produção da alface não é diferente, como mostra no Gráfico 1, de acordo com os dados coletados no site do Ceasa de Campinas durante o 20 semestre de 2015.

Gráfico 01. Analise de Variação de Resultado da Alface no 2올 Semestre de 2015.

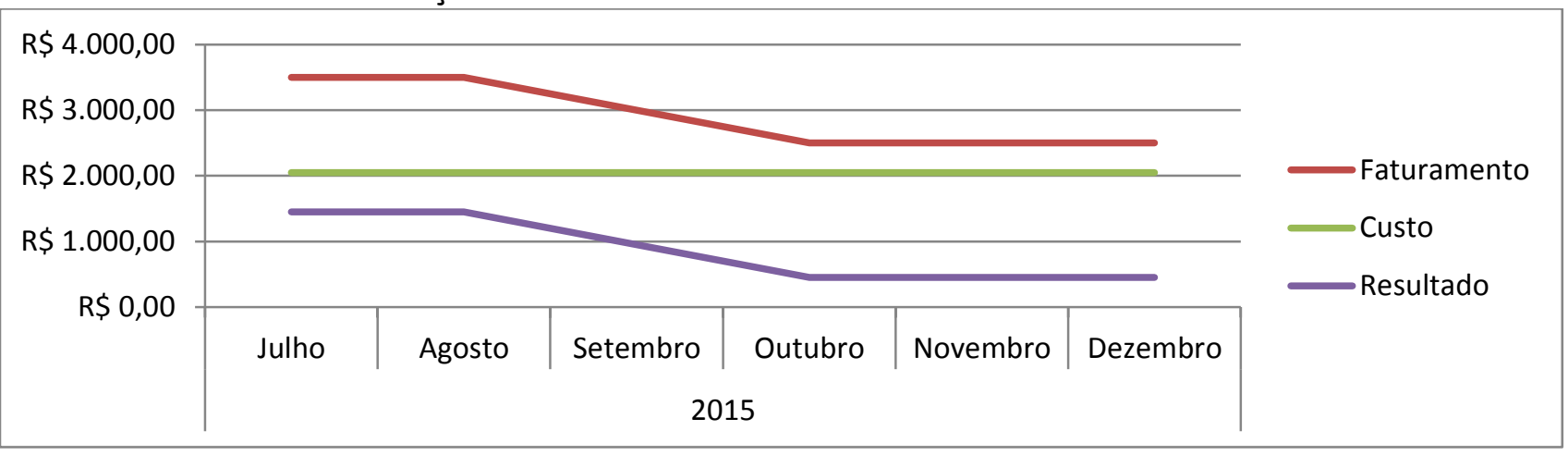

Nos meses de julho e agosto de 2015, segundo dados de preços de mercado do Ceasa de Campinas, o faturamento por hectare da alface produzido seria de $\mathrm{R} \$ 3.499,99$, no mês de setembro de $R \$ 2.999,99$, e nos meses de outubro, novembro e dezembro, de $R \$ 2.499,99$. Ou seja, o produtor teria um faturamento nos meses em questão de $\mathrm{R} \$ 17.499,93$.

Por meio dessa analise, percebe-se uma diferença significativa de preços. Deve o produtor planejar-se para vender as maiores quantidades nos meses onde o preço de mercado seja melhor.

Os custos ao longo do ano permaneceram estáveis, sendo uma média de $\mathrm{R} \$ 2.049,51$, por hectares, perfazendo um montante de $\mathrm{R} \$ 12.297,05$. O resultado da produção oscilou de acordo com a oscilação dos preços de mercado da alface, sendo que, nos meses de julho e agosto, um total de $\mathrm{R} \$ 1.450,48$ e no mês de setembro $\mathrm{R} \$ 950,48$. No trimestre outubro, novembro e dezembro foi de $\mathrm{R} \$ 450,48$, totalizando $\mathrm{R} \$ 5.202,88$.

No segundo semestre de 2016, de acordo com o Ceasa de Campinas, também ocorreu oscilação no preço pago ao produtor, como demonstrado no Gráfico 2. No mês de janeiro de 2016, o faturamento por hectare seria de $\mathrm{R} \$ \mathbf{2 . 4 9 9 , 9 9}$, no mês de fevereiro, de $\mathrm{R} \$ 3.333,32$, março de $R \$ 4.166,65$, abril de $R \$ 4.333,32$. No mês de maio, de $R \$ 5.133,31$ e o mês de Junho, de $R \$$ $5.749,98$.

Gráfico 02. Analise de Variação de Resultado do Alface no 1옹 Semestre de 2016.

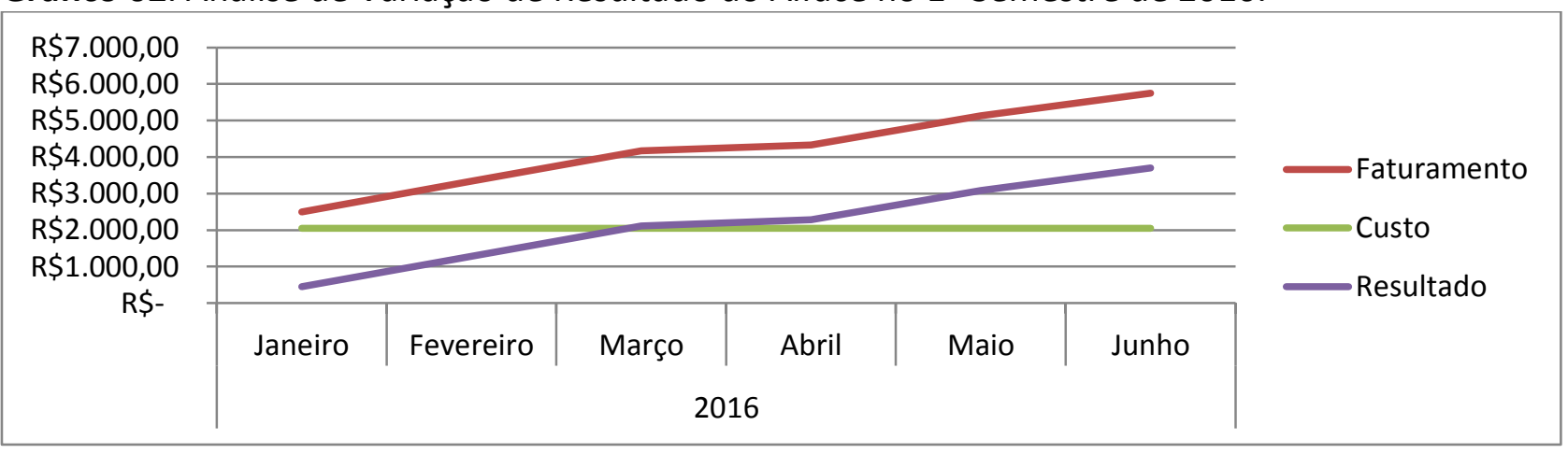

O custo, assim como no 2 o semestre de 2015 , não oscilou, manteve a média de $\mathrm{R} \$ \mathrm{R} \$$ $2.049,51$, totalizando no semestre, $\mathrm{R} \$ 12.297,05$. Já o resultado acompanhou a oscilação do faturamento sendo no mês de janeiro $R \$ 450,48$, no mês de fevereiro, $R \$ 1.283,81$, março $R \$$ $2.117,14$, abril $R \$ 2.283,81$, em maio, de $R \$ 3.083,80$ e por fim, no mês de junho, $R \$ 3.700,47$, totalizando no 1 - semestre de $2016, \mathrm{R} \$ 12.919,51$.

Sendo assim, dentro de um ano de produção, o faturamento seria de $R \$ 42.716,50$, os custos $\mathrm{R} \$ 24.594,10$ e o resultado anual da produção de $\mathrm{R} \$ 18.122,39$. 


\section{DISCUSSÃO}

O custo de produção da alface por hectare é formado por $52 \%$ de custos com insumos e $48 \%$ de serviços. A cultura sofre os efeitos da sazonalidade do mercado, ou seja, ocorre durante o ano oscilação no preço de mercado, impactando os resultados financeiros.

A elaboração de uma planilha permite ao produtor observar a composição dos custos, a oscilação dos preços de mercado bem como os resultados obtidos e ainda promover intervenções para melhorar o desempenho financeiro da atividade.

Como alternativa para melhorar o custo de produção, a adubação orgânica, de acordo com a analise de solo, pode ser substituída por esterco bovino ou compostagem que são historicamente insumos mais baratos.

Outra opção para a redução de custos pode ser feita com desbastes, capinas e adubação de cobertura, que representam $22,24 \%$ dos custos com insumos. Neste caso, a utilização de palhada sobre os canteiros, evita o surgimento de plantas invasoras, dispensando as capinas, e ainda reduz a necessidade de adubação de cobertura. Essa pratica, quando possível, proporciona redução dos custos com insumos com reflexos positivos nos resultados.

Segundo Matos (2008), os melhores preços são pagos aos produtores em meses quentes e úmidos e os menores preços ocorrem nos meses secos e frios. Essa situação é provocada pela redução de produção da alface devido aos fatores climáticos que dificultam a produção da mesma, ou seja, altas temperaturas e alta umidade. O inverso é provocado pelo aumento da produção devido aos fatores climáticos que favorecem a produção, como baixas temperaturas e baixa umidade.

\section{CONCLUSÃO}

A cultura da alface, conforme demonstrado, sofre oscilações de preços de mercado ao longo do ano, em contrapartida os custos mantiveram-se relativamente estáveis e, mesmo diante das alterações de mercado, apurou-se lucratividade de $42,42 \%$ para um hectare no período de um ano. Para obter resultados satisfatórios, é preciso desenvolver eficiência produtiva, gestão de negócio e conhecimento dos indicadores de mercado.

O estudo em questão permitiu evidenciar que a gestão financeira e o controle sobre as operações são fundamentais para a sobrevivência do negócio no médio e longo prazo e, não menos importante, a utilidade das informações e indicadores para que o produtor possa tomar decisões sobre novos investimentos, cortes de custos e procurar por novos mercados consumidores. Assim sendo, o produtor deve orientar-se por práticas econômicas, financeiras, e administrativas que visam a sustentabilidade da cultura bem como da propriedade, preservando o meio ambiente e garantindo a manutenção dos recursos naturais para futuras gerações.

\section{REFERÊNCIAS}

CEASA Campinas, Conotações Anteriores. Preços. Disponível em: <http://www.ceasacampinas.com.br/novo/cotacao_anterior.asp>, Acessado em 20 de Julho de 2016.

COSTA, C. P.; SALA, F. C. A evolução da alfacicultura brasileira. Horticultura Brasileira, Brasília, v. 23, n. 1, 2005.

IEA. Técnicas Agropecuárias. Instituto Educacional Aprovação. Santa Cruz do Sul: IEA, 2005.

RESENDE, F. V.; SAMINÊZ, T. C. O.; VIDAL, M. C.; SOUZA, R. B.; CLEMENTE, F. M. V. Cultivo de Alface em Sistema Orgânico de Produção. Circular Técnica. Brasília: Embrapa Hortaliças, 2007. 
MATOS, F. A. C; JÚNIOR, A. D. C.; SERRA, D. D.; BOAVENTURA, E. C.; DIAS, R. L.; CASCELLI; S. M. F. Alface: Saiba como cultivar hortaliças para colher bons negócios. Série Agricultura Familiar. Brasília: Sebrae, 2008.

SEDIYAMA, M. A. N.; RIBERO, J. M. O.; PEDROSA, M. W. Alface. In: JÚNIOR, T. J. P.; VENZON, M. 101 culturas: manual de tecnologias agrícolas. Belo Horizonte: EPAMIG, 2007. p. $53-62$.

SOUZA, B. F.; SCHLOTTFELDT, D. P.; FONSECA, H. N. P.; FALCÃO, J. V.; NETO, S. A. R. Custo de Produção Agrícola: A metodologia da Conab. Companhia Nacional de Abastecimento. Brasília: Conab, 2010. 